\title{
Orkney's approach to COVID-19
}

\section{By Alice Bowen}

$\mathrm{U}$

nique to the Orkney islands, an archipelago composed of 70 islands of which 20 are inhabited, has been the co-ordination and testing at multiple remote locations in response to COVID-19.

Challenges arose where island resources and settings are not identical, nor the medical professional based on each respective island. This made it essential to think laterally and develop a protocol which was adaptable to remote communities, but crucially maintained decontamination of the samples and timely processing at the labs. Starting with Orkney's largest island, 'Mainland', a swabbing protocol was developed and troubleshot, at the local COVID assessment centre, by the team of voluntary dentists and dental professionals. Video resources of the procedure were filmed, developed and sent to remote communities as a training resource to establish a standardised approach to swabbing.

Redeployment of dentists in our remote setting was a necessary step to support the testing of our populace. Considering the swabs were taken from the oropharynx and nasopharynx, this was a task well suited to dental professionals, given our anatomy teaching in addition to our familiarity with cross

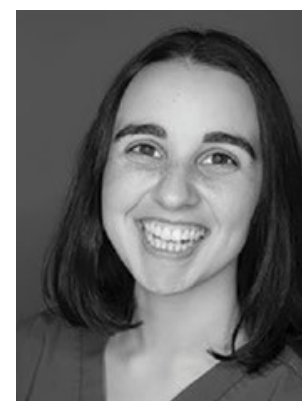
contamination, PPE and consent.

Positive outcomes for our dental team have been the close interaction and training with our medical colleagues as part of the COVID-19 response. Training has included specific COVID-19 history taking, decision making and case presentation: a format we are well used to. As a hub this medico-dental interface led to the creation of an assessment template, later sent to colleagues working in Tayside and Fife.

Resumption of normal dental practice is an unclear picture at present; redeployment has however equipped us with knowledge regarding the presentation of COVID-19, familiarity with remote consultations and a stronger, more personal working relationship with our medical colleagues.

As a recent dental graduate, moving to the Orkney Islands to take up a VT post and now working as part of the COVID-19 response team were certainly unforeseen circumstances some 12 months ago. Although not the standard VT year, redeployment has benefitted our dental team, working more closely together, as well as the connection with our community in these uncertain times. 